\title{
The emerging role of magnetic resonance imaging and multidetector computed tomography in the diagnosis of dilated cardiomyopathy
}

\author{
Massimo Slavich • Anca Florian • Jan Bogaert
}

Received: 21 December 2010 /Revised: 25 February 2011 / Accepted: 2 May 2011 / Published online: 19 May 2011

(C) European Society of Radiology 2011

\begin{abstract}
Magnetic resonance imaging and multidetector computed tomography are new imaging methods that have much to offer clinicians caring for patients with dilated cardiomyopathy. In this article we briefly describe the clinical, pathophysiological and histological aspects of dilated cardiomyopathy. Then we discuss in detail the use of both imaging methods for measurement of chamber size, global and regional function, for myocardial tissue characterisation, including myocardial viability assessment, and determination of arrhythmogenic substrate, and their emerging role in cardiac resynchronisation therapy.
\end{abstract}

Keywords Dilated cardiomyopathy $\cdot$ Magnetic resonance imaging $\cdot$ MRI $\cdot$ Multidetector computed tomography · MDCT

\section{Introduction}

The term "cardiomyopathy" identifies a heterogeneous group of cardiac diseases characterised by direct myocardial involvement leading to impaired cardiac function. Since the term was introduced by Harvey and Brigden in the 1960s, substantial progress has been made, in terms of understanding the pathophysiological substrate, underlying causes and peculiarities, and most importantly, in defining a

Electronic supplementary material The online version of this article (doi:10.1007/s13244-011-0101-8) contains supplementary material, which is available to authorized users.

M. Slavich · A. Florian · J. Bogaert $(\bowtie)$

Department of Radiology and Medical Imaging Research Center,

UZ Leuven,

Herestraat 49,

3000 Leuven, Belgium

e-mail: Jan.bogaert@uz.kuleuven.ac.be classification system for cardiomyopathies. Primary cardiomyopathies include those with genetic, acquired or mixed causes, and refer to a disease that is predominantly limited to the myocardium, whereas secondary cardiomyopathies are characterised by myocardial involvement that is part of a generalised or diffuse systemic disorder. In addition, several cardiovascular diseases not regarded as cardiomyopathies may also affect the myocardium, causing systolic or diastolic dysfunction and hampering differentiation from heart muscle diseases [1]. Although with the current arsenal of diagnostic imaging tools, the diagnosis of cardiomyopathies has been facilitated, in many cases the correct diagnosis remains challenging and sometimes elusive. Nevertheless, these techniques have significantly contributed towards an increased awareness of cardiomyopathies among clinicians, and they have an increasing impact on patient management and risk stratification. Transthoracic echocardiography is currently the first-line imaging technique for the diagnosis, evaluation and decision-making in cardiomyopathy patients. In the last decade, magnetic resonance imaging (MRI) and, to a lesser extent, multidetector (or multirow) computed tomography (MDCT) have become increasingly important in the diagnosis of cardiomyopathies. In particular, the comprehensive approach of MRI, including non-invasive tissue characterisation, makes it indispensable in the work-up of many cardiomyopathies.

\section{Dilated cardiomyopathy}

Dilated cardiomyopathy (DCM) is the most common cardiomyopathy worldwide with a prevalence of $40-50$ cases per 100,000. DCM is currently classified as primary (genetic, acquired or mixed), secondary (e.g. infiltrative or 
autoimmune) and idiopathic. Genetic inheritance is likely to play a role in the development of the disease in $20-35 \%$ of patients, but also acquired conditions like metabolic abnormalities, inflammatory and infectious processes, neuromuscular diseases and a large variety of cardiotoxic agents (chemotherapeutic agents, alcohol, illicit drugs) can lead to DCM [1-4]. Independently of the underlying cause, DCM is characterised by an increase in diameter and volume of the left or both ventricles, leading to progressive dilatation and impaired systolic function that is not secondary to or cannot be exclusively justified by abnormal loading conditions (e.g. valve disease, hypertension) or by the concomitant coronary artery disease (CAD) (Figs. 1 and 2) (Movie 1) [1-3]. Thus, the term DCM represents a final common pathway that is the end result of myocardial damage.

\section{Pathophysiological and histological aspects}

Independently of the underlying cause, DCM is characterised by an increase in ventricular chamber size. As demonstrated by the Frank-Starling mechanism, this serves as a first compensatory step aiming to maintain an appropriate stroke volume. However, above a critical sarcomere stretch, the efficiency of interaction between actin and myosine filaments decreases, resulting in impairment of stroke volume. Myocardial fibre elongation increases the ventricular radius, causing eccentric ventricular hypertrophy, with decreased wall thickness to chamber diameter ratio and increased ventricular sphericity. According to the Laplace law, these changes increase significantly myocardial wall stress with increased oxygen demand and subsequent worsening of the left ventricular (LV) systolic performance [5]. The main histological features of DCM are myocyte elongation, myocardial apoptosis and hypertrophy of the remaining myocytes [6]. Additionally, there is an excessive collagen deposition and decreased capillary density, with both reactive (interstitial and perivascular) and reparative (replacement) patterns of fibrosis [7, 8]. Myocardial fibrosis is considered the result of damage due to microvascular ischaemia and myocardial wall inflammation. The cellular and extracellular changes result in a normal, thinned or slightly thickened myocardial wall (Fig. 1).

\section{Clinical manifestation}

The clinical presentation of DCM is variable, but the severity of symptoms is usually related to the grade of systolic impairment. Although patients can be asymptomatic, especially in the early phases of the disease, most of them have symptoms of left heart failure, such as dyspnoea and effort-related fatigue. In some cases, severe LV systolic dysfunction and advanced heart failure symptoms are present in patients with normal or mildly dilated ventricles (less than $10-15 \%$ above normal range), i.e. mildly dilated forms of DCM [1]. Sudden cardiac death can be the first clinical evidence of DCM and it is due to sustained ventricular tachycardia and ventricular fibrillation. It is deemed that the more severe the systolic dysfunction and the degree of replacement fibrosis, the larger the arrhythmogenic substrate that can provoke malignant arrhythmias. Other potentially life-threatening manifestations of DCM are non-sustained ventricular arrhythmias, conduction abnormalities, syncope and embolic events. As the disease progresses, right heart failure may develop, and it is considered an aggravating factor (Fig. 3) [9, 10]. Thus, right ventricular (RV) dilatation and dysfunction can be present, but are not requirements for the diagnosis of DCM.
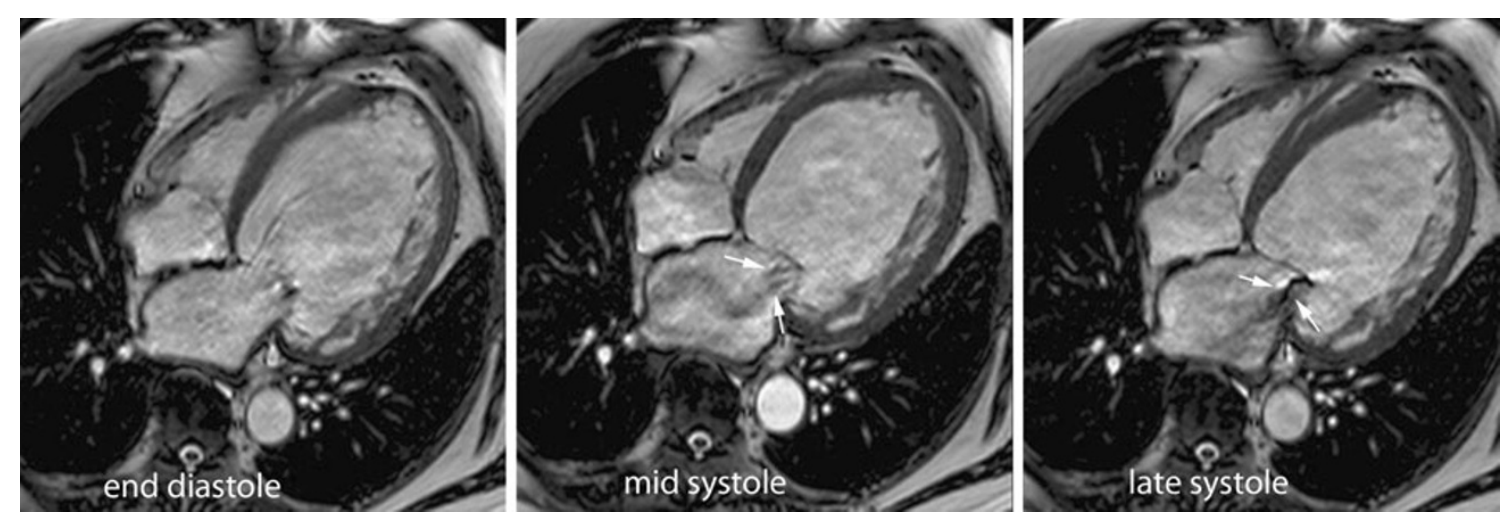

Fig. 1 Extreme form of dilated cardiomyopathy in a 63-year-old man. SSFP cine MRI in a four-chamber view at end-diastole (left), midsystole ( $m i d$ ), and late-systole (right) (see Movie 1). The left ventricle (LV) end-diastolic volume is $877 \mathrm{ml}$ with an ejection fraction of $12 \%$. The right ventricle (RV) end-diastolic volume is $248 \mathrm{ml}$ with an ejection fraction of $42 \%$. Presence of an important mitral regurgitation secondary to dilatation of the mitral valve ring. The regurgitation is visible during systole (white arrowheads in the middle and right panels). Velocity-encoded MRI shows a regurgitant volume of $45 \mathrm{ml}$ through the mitral valve 
Fig. 2 LV volume overload due to aortic regurgitation in 21year-old man with Marfan's MR image in a three-chamber view (a) and LV outflow tract view (b). Velocity-encoded cine aortic valve at systole (c) and diastole (d). Dilated LV with an end-diastolic volume of $500 \mathrm{ml}$, a stroke volume of $255 \mathrm{ml}$ and an ejection fraction of $51 \%$. Cine MRI (a, b) shows important aortic regurgitant jet (arrows). Velocity-encoded cine MRI shows non-closure of the aortic valve leaflets during fraction of $43 \%$ disease. Diastolic SSFP cine MRI just below the level of the diastole (d) with a regurgitant
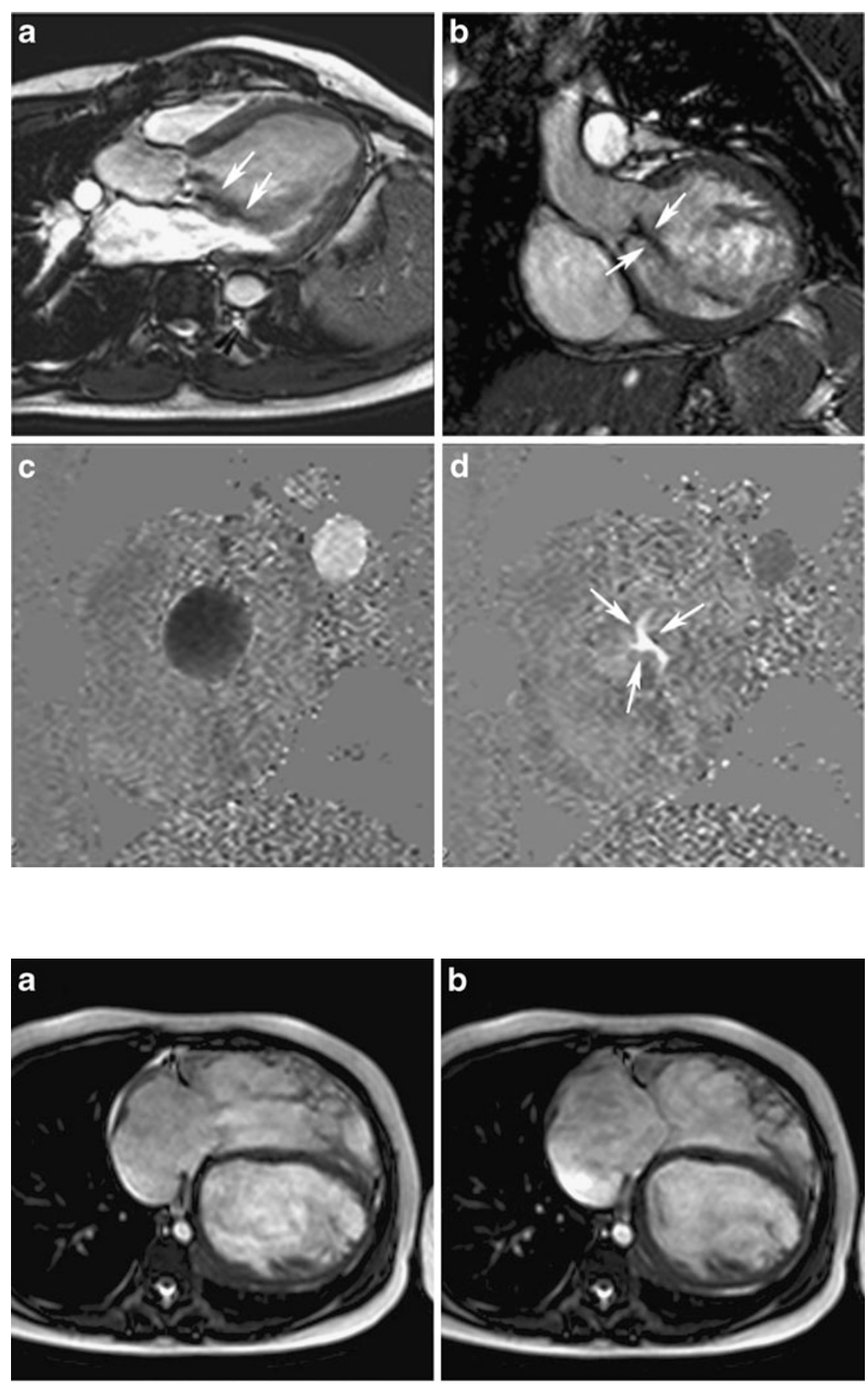

Fig. 3 Extreme form of dilated cardiomyopathy in an 11-yearold girl involving both ventricles, and clinically presenting with severe symptoms of heart failure. SSFP cine MR image in the axial plane at end-diastole (a) and end-systole (b). Late gadolinium enhancement (LGE) MRI in the midventricular short-axis plane (c) and axial plane (d). Note the important dilatation of both ventricles, i.e. $\mathrm{LV}$ end-diastolic volume of $324 \mathrm{ml}, \mathrm{RV}$ end-diastolic volume of $520 \mathrm{ml}$ with an ejection fraction of $9 \%$ and $7 \%$ for the LV and RV respectively. LGE MRI shows diffuse strong subepicardial enhancement in the LV (white arrows in $\mathbf{c}, \mathbf{d}$ ), along the subendocardium of the RV (black arrows) (d), and of the RV free wall (white arrowheads) (d). Unfortunately the patient died while on the waiting list for a cardiac transplant
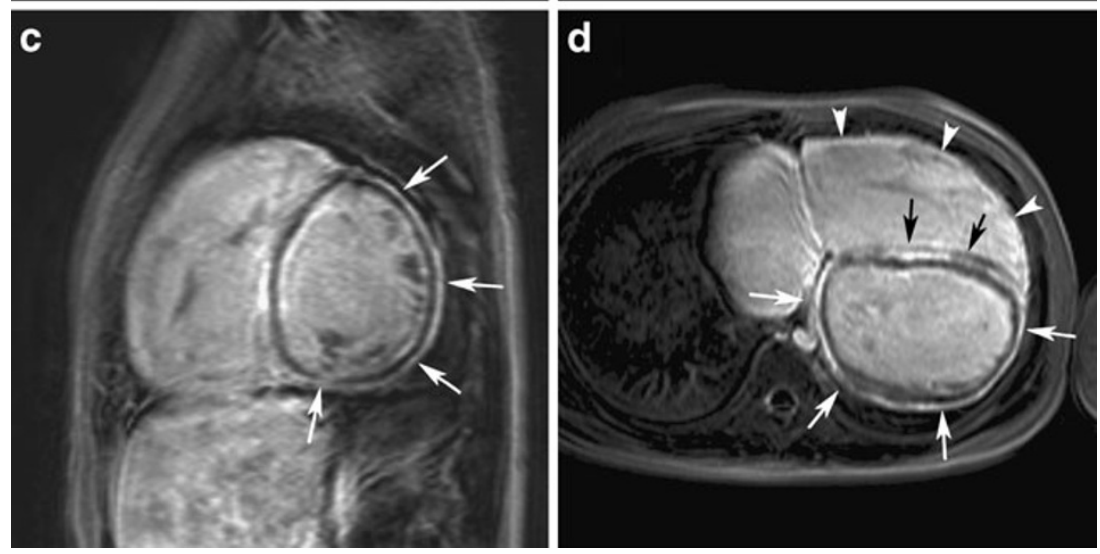


\section{MRI and MDCT}

The requirements for appropriate imaging in cardiomyopathies nowadays exceed the pure diagnostic objective, whereby assessment of the more intricate aspects, such as depiction of underlying aetiology, risk assessment, therapy planning and determination of prognosis, are becoming increasingly important. MRI, because of its non-invasive nature, its excellent spatial, contrast and temporal resolution, and capability for tissue characterisation, has become a well-validated and widely accepted technique for studying DCM patients. In addition, the constant improvement in MDCT technology, especially since the introduction of the 64-row systems, offers reliable imaging of the heart and coronaries, offering new, yet incompletely explored approaches to studying DCM patients (Fig. 4). Several studies have independently shown that MDCT is highly appropriate for excluding coronary artery disease, yielding excellent negative predictive values [1114]. Moreover this technique enables the characterisation of the coronary venous system, which may be of great help in the planning of cardiac resynchronisation therapy (CRT). In patients with contra-indications for MRI, such as those with permanent cardiac implantable devices, MDCT is an interesting diagnostic alternative.

Cine MRI is nowadays considered the reference technique for accurately measuring cardiac chamber size, ventricular function and myocardial mass (Figs. 1 and 3) [10, 15-18]. Moreover, derived parameters, such as
Fig. 4 Ventricular assist device in a 62-year-old man with endstage DCM. MDCT showing four axial slices through the heart and upper thorax (a-d), volume-rendered view (e). Note the presence of a severely dilated LV with thin walls. The LV is shortcut by the assist device between the left atrium and right subclavian artery (arrow in b, d). The course of the ventricular assist device can be well appreciated on the volume-rendered view (arrowheads in e)
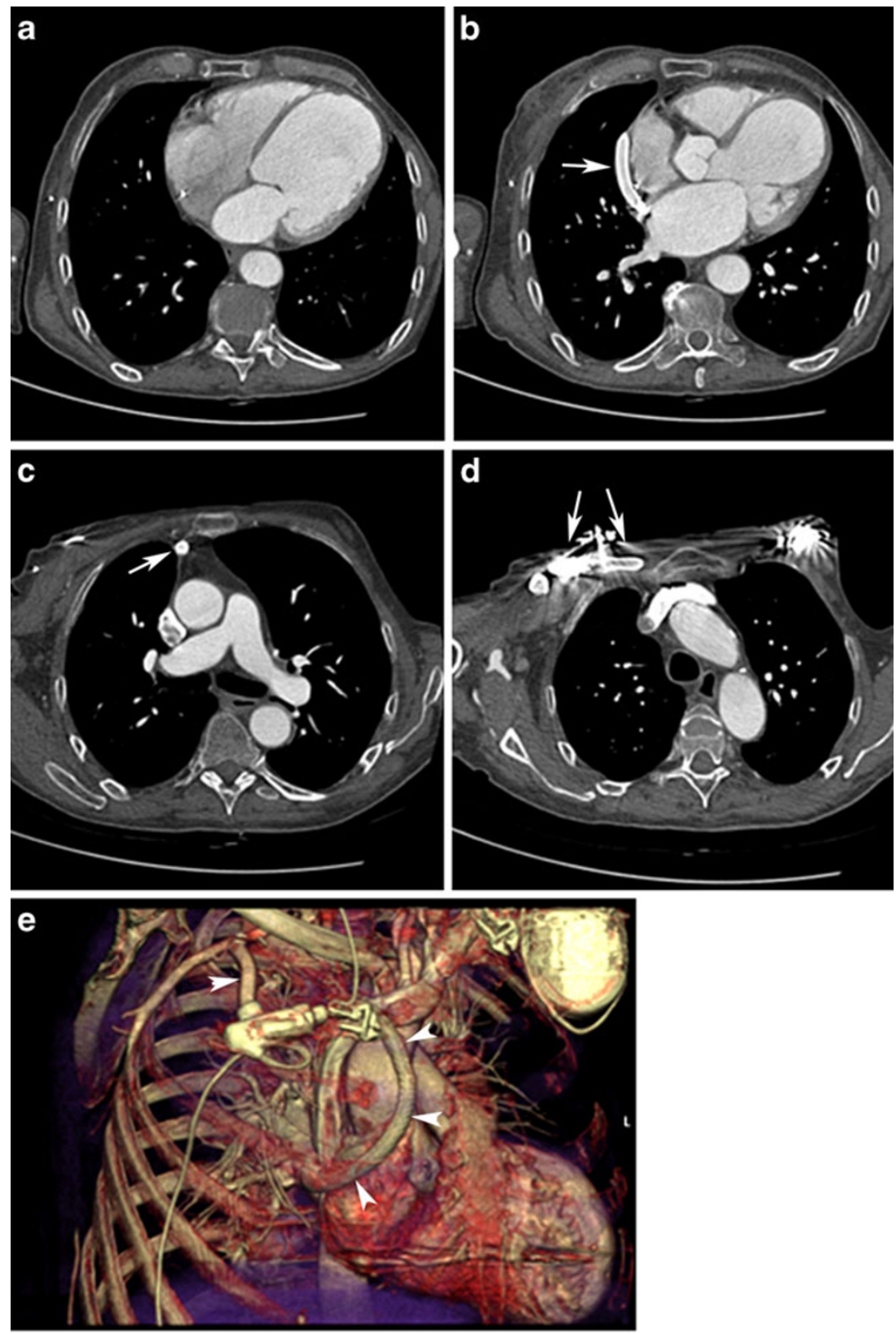
myocardial wall thickness, wall thickness to chamber radius ratio and regional functional parameters (e.g. systolic wall thickening and wall motion), can be reliably assessed. DCM-related pathological conditions such as thrombus formation or valve dysfunction can be depicted by cine MRI as well. Techniques such as myocardial tagging have been shown to be very helpful in understanding the altered myocardial deformation patterns in DCM patients $[19,20]$. MRI can be used for myocardial tissue characterisation, using T2-/T2*-weighted sequences and/or contrastenhanced MRI. These sequences may even replace endomyocardial biopsy in some diseases (e.g. iron deposition cardiomyopathy). T2-weighted sequences allow increased myocardial free water content to be depicted, which occurs in the setting of acute myocardial infarction or in other diseases characterised by inflammation, such as acute myocarditis. Affected regions will appear bright compared with the normal myocardium. In the case of diffuse myocardial oedema, some authors recommend to measure the difference in signal intensity between myocardium and skeletal muscle (Fig. 5) [21]. T2*-weighted sequences are of great value for the diagnosis of myocardial iron deposition diseases, leading to a shortening of $\mathrm{T} 2 *$ relaxation time (Fig. 6). This parameter can be used to predict complications such as heart failure and arrhythmias and can be used to initiate and monitor chelation therapy $[22,23]$. Compared with normal myocardium, in pathological myocardium the gadolinium contrast kinetics are altered, secondary to changes in distribution volume and/ or wash-out of gadolinium. For the diagnosis of focal myocardial disease, such as necrosis/fibrosis, contrastenhanced imaging using fast gradient-echo sequences with a preparatory inversion pulse [the so-called "late gadolinium enhancement" (LGE) or "delayed enhancement" technique] have been shown to be of great value [24]. Although initially applied to depict myocardial necrosis/ fibrosis in the setting of acute or chronic myocardial infarction, the LGE MRI technique allows several nonischaemic patterns of myocardial enhancement to be described in a wide range of myocardial diseases [25]. A weakness of the LGE MRI technique, however, is that it is optimised for depicting focal myocardial pathological conditions, and therefore it may fail to show diffusely spread disease, such as diffuse myocardial fibrosis, found in patients with DCM [26]. Comparing global myocardial enhancement with skeletal muscle enhancement early after gadolinium administration is of use to depict generalised myocardial hyperaemia, which can be found in patients with myocardial inflammation not only in the acute phase but also in chronic forms of myocarditis (Fig. 5) [27, 28]. Besides, myocardial T1 mapping techniques are appealing for the depiction of diffuse myocardial fibrosis, and represent a valuable addition to the LGE MRI technique. Shortening of the myocardial T1 relaxation time is related to the amount of myocardial collagen deposition [26, 29, 30]. Performing fast gradient-echo sequences using multiple increasing inversion times (e.g. 50-1,000 ms) before and after contrast-medium administration at the blood/ myocardium equilibrium phase, allows the decay in myocardial signal intensity to be measured, and T1 maps to be generated with the use of curve fitting techniques (Movie 2). Although this technique is promising, further investigation is needed to test its robustness in assessing patients in mainstream clinical practice. Finally, a free
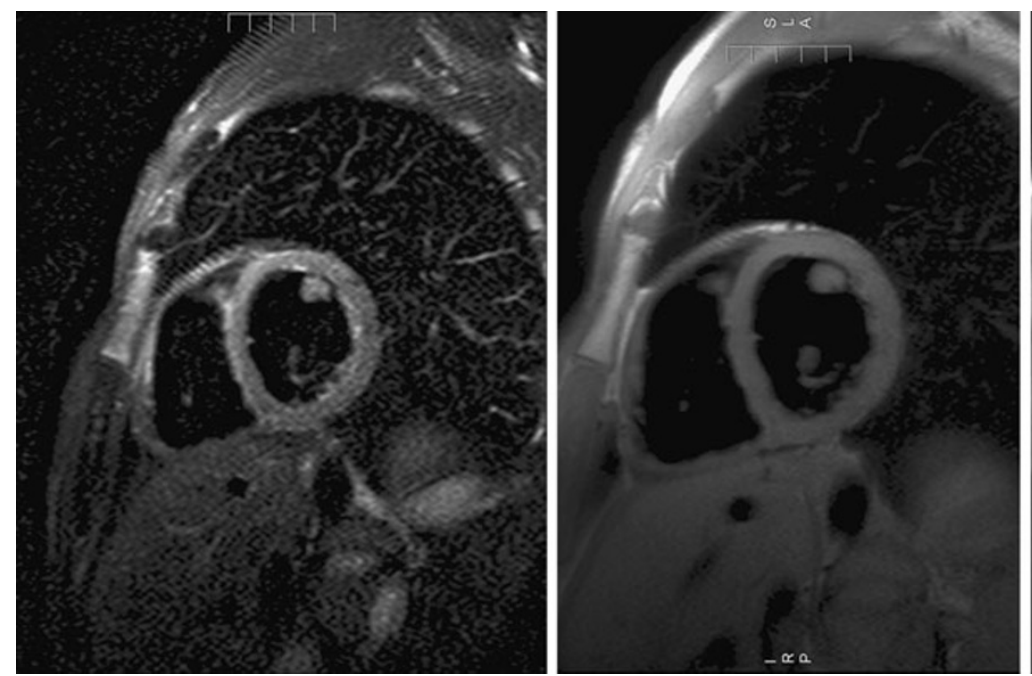

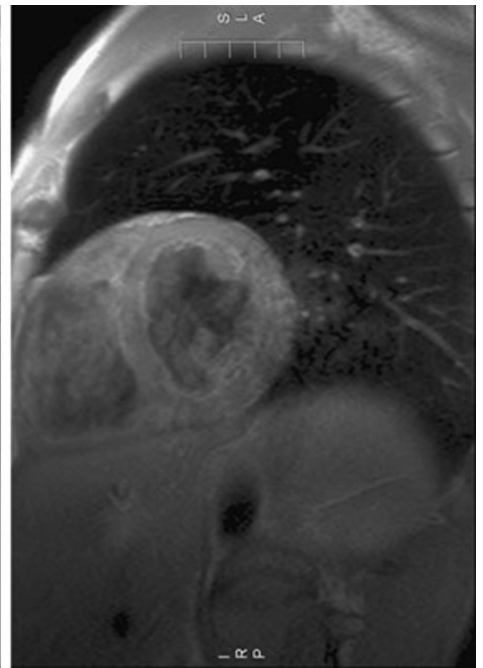

Fig. 5 MRI approach for depiction of changes in global myocardial tissue characteristics. Midventricular short-axis T2-weighted short inversion-time inversion-recovery (STIR) fast spin-echo (left), T1weighted fast spin-echo before (middle) and at 4 min after administration of $0.2 \mathrm{mmol}$ of gadolinium DTPA $/ \mathrm{kg}$ body weight (right).
Calculation of the ratio of the differences in signal intensity between myocardial and skeletal muscle using T2-weighted STIR fast spinecho allow generalised myocardial oedema to be depicted (left), while the changes in signal intensity early after contrast medium administration allow myocardial hyperaemia to be visualised (right) 


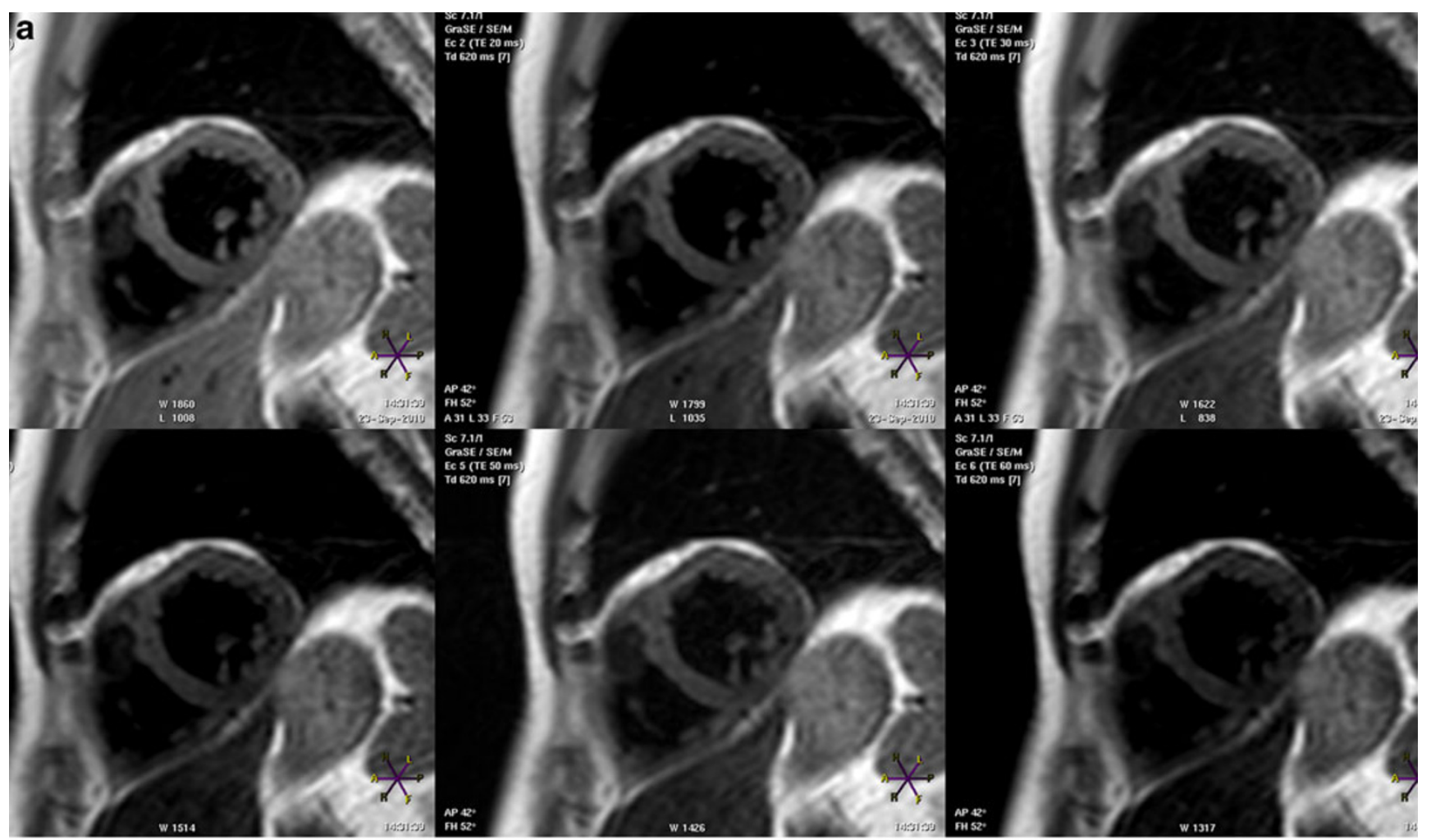

\section{b}

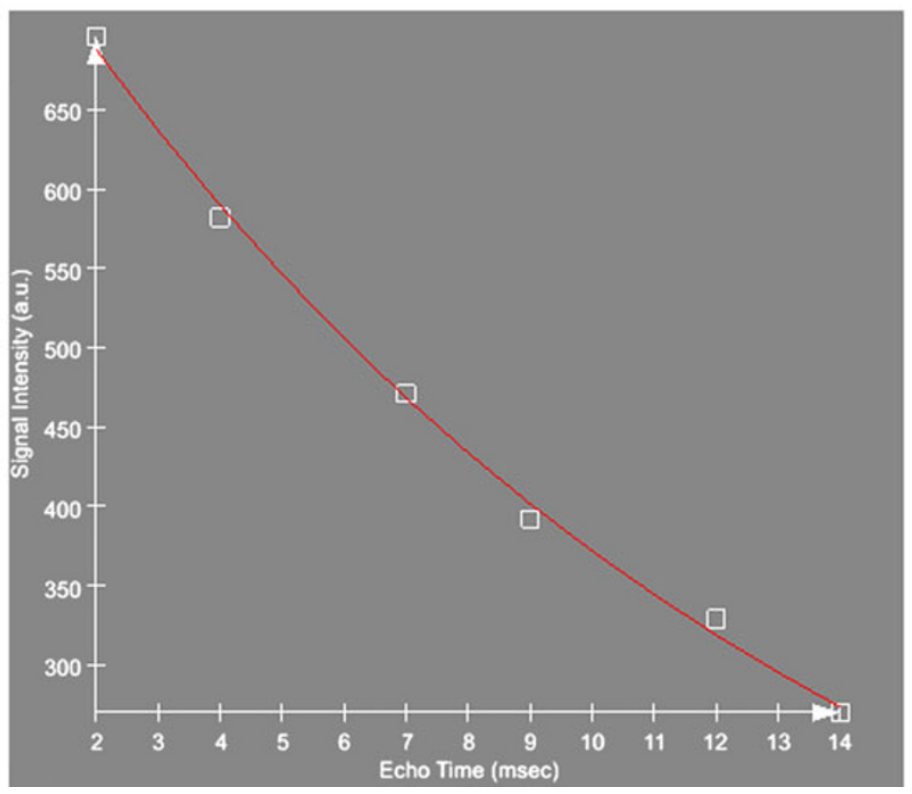

Fig. 6 Hereditary haemochromatosis in a 56-year-old man. T2*-weighted sequences show significantly decreased myocardial $\mathrm{T} 2 *(13 \mathrm{~ms})$ (normal value at 1.5 Tesla: $33.3 \pm 7.8 \mathrm{~ms}$ ). The patient showed no evidence of systolic or diastolic dysfunction

bonus of the LGE MRI technique is that concomitant cardiac disease, such as pericardial inflammation or thrombus formation, can be accurately depicted as well [31-33].

Even if MDCT is not yet considered as a first-line imaging technique for the evaluation of LV performance and volumes, it is important to mention that in patients undergoing an MDCT examination for coronary artery evaluation, reliable information about cardiac morphology and function can be obtained without any additional radiation exposure [34-37]. Although nowadays the prospective trigger mode is preferable because of the signifi- 
cant reduction in irradiation dose, reliable data regarding ventricular volumes and function can be obtained using retrospective electrocardiogram (ECG) gating. Several papers reported good agreement among MDCT and echocardiography, and MRI and invasive catheter ventriculography, with good interobserver agreement [38-41].

\section{Cardiac volumes and ventricular function}

As the LV ejection fraction is the strongest prognostic determinant in heart failure patients, while LV volume and mass are independent predictors of mortality and morbidity, a first primordial step in assessing DCM patients is the reliable quantification of the severity of chamber dilatation and dysfunction. Often stroke volumes are within normal limits or only modestly decreased despite the severely impaired ejection fraction. The LV enlargement may furthermore dilate the mitral valve ring, dislocate the papillary muscles, and impair leaflet coaption, thereby causing mitral valve regurgitation and putting additional load on the already diseased ventricle (Fig. 1). Except for mildly dilated forms of DCM, the LV and/or RV show a moderate to severe degree of dilatation with a severely impaired ejection fraction (e.g. lower than 20\%) (Figs. 1 and 3). The volumetric measurement of the ventricles is usually performed in the cardiac short-axis plane (MRI) or using reconstructed images in the cardiac short-axis plane (MDCT). For MDCT evaluation of LV function, enddiastole is usually identified as the image with the maximum diameter (approximately at $85 \%$ of RR diameter), while the minimum diameter (about $25 \%$ of RR) corresponds to end-systole. Software has been developed to semi-automatically determine ventricular volumes and function, and myocardial mass.

In DCM patients, the atria may be enlarged as well, but this is usually less pronounced than the ventricular dilatation. Left atrial dilatation can be related to mitral valve regurgitation and/or to increased LV filling pressure, emphasising the need for assessment of diastolic (dys) function in DCM patients. Phase-contrast (or velocityencoded) cine MRI is an accurate technique for quantifying the severity of valve regurgitation and for providing information on diastolic function. Although MDCT can provide MR-equivalent high-quality morphological views of the mitral valve, and even show valve motion, it provides no or at most limited information regarding the severity of regurgitation. However, the superb morphological visualisation of the mitral valve and subvalvular apparatus provide a means of better characterising the mechanism(s) of mitral valve regurgitation. Moreover, slow or turbulent flow in dilated cavities facilitates thrombus formation. Both MRI and MDCT can be regarded as excellent tools for depicting this potentially harmful complication (Fig. 7).

\section{Myocardial contractility}

Myocardial contraction abnormalities are invariably present in DCM patients, visible as hypokinetic to dyskinetic wall motion, diminished to absent systolic wall thickening, and a variable degree of ventricular dyssynchrony often with abnormal systolic motion of the interventricular septum and apical rocking (Movie 3). Cine MRI is without any doubt the reference tool for assessing myocardial wall motion and thickening patterns, and has potential in the assessment of ventricular dyssynchrony. Regional abnormalities are usually described using the 17-segment AHA approach.

\section{Myocardial tissue characterisation}

Accurate myocardial tissue characterisation is pivotal in DCM patients. For example, as reported by Bello et al. [42], the response to b-blockade therapy was significantly better with functional improvement and reversed ventricular remodelling in heart failure patients without evidence of myocardial scarring by LGE MRI than those with myocar-
Fig. 7 Small thrombus in the LV apex in a male patient with a moderately dilated cardiomyopathy. Vertical long-axis SSFP cine MRI (left), contrast-enhanced MRI early after contrast medium administration using a long inversion time (i.e. $550 \mathrm{~ms}$ ) (right). The thrombus is hardly visible on cine MRI (left) but is well depicted as a hypointense rounded nodular structure on the contrast-enhanced image (right)

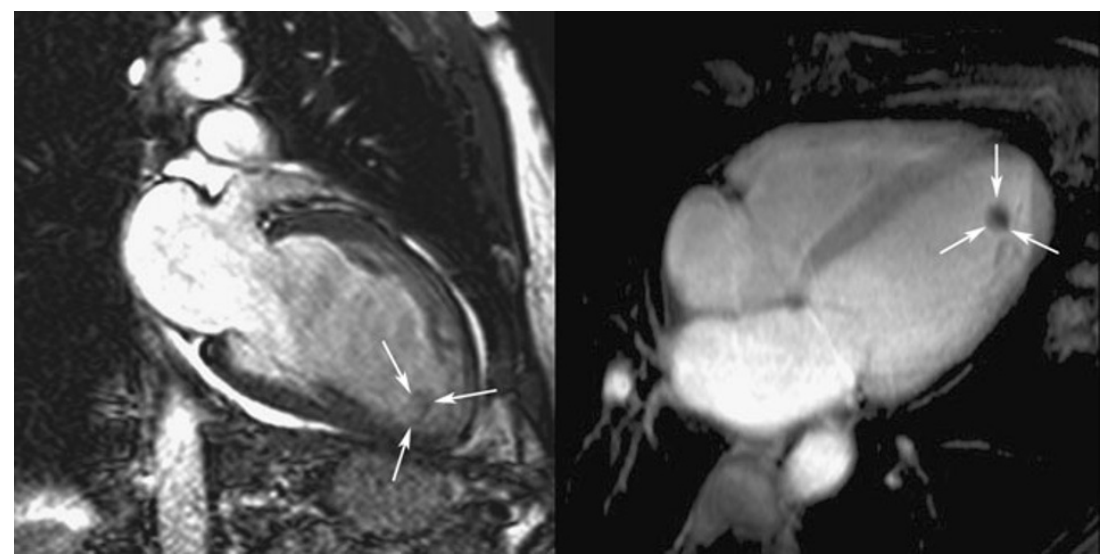


dial scarring. A first aim is to exclude myocardial damage due to $C A D$ as the underlying cause of ventricular dilatation and dysfunction [43-45]. The presence and pattern of myocardial LGE provides crucial information regarding the ischaemic or non-ischaemic origin. Ischaemic LGE involves the subendocardium with a variable transmural extension in a coronary artery perfusion territory (Fig. 8). In non-CAD-related DCM, myocardial LGE presents most commonly as a linear or patchy midwall enhancement. It may involve the subepicardial part of the myocardium and the right ventricle as well, and importantly it does not respect a perfusion territory (Fig. 3) [44-46]. It should be emphasised, however, that most DCM patients present no myocardial LGE [45]. Finally, a small percentage of DCM patients show a CAD-like pattern of enhancement, despite a lack of obstructive CAD on coronary angiography. A likely hypothesis is that these patients had concomitant CAD with recanalisation of the occlusive coronary artery or had an embolic event. Ventricular remodelling and dysfunction will be influenced significantly by the extent of CAD-related myocardial scarring. Although use of MDCT has been reported to depict CAD-related myocardial scarring in chronic infarct patients, currently this application is not routinely used [47].

Although LGE MRI is able to depict subtle forms of myocardial scarring $(<1 \mathrm{~g})$ [48], this sequence is of limited value in depicting diffuse myocardial fibrosis, probably explaining why most DCM patients in the study by McCrohon et al. [45] showed normal LGE MRI. Recently, substantial progress has been made with the development of myocardial T1 mapping techniques. Diffuse collagen deposition increases the extracellular space, causing an increased interstitial accumulation of gadolinium at steady state, thus reducing the myocardial T1 relaxation time [26, 29, 30, 49, 50]. Several groups have reported in DCM patients a tight relation between the expansion in extracellular space (reflecting myocardial fibrosis) and the impairment in myocardial blood flow, ventricular dilatation and ventricular dysfunction [29, 50-52].

It is believed that approximately $5-10 \%$ of patients with acute myocarditis progress towards DCM and ultimately will need cardiac transplantation $[1,53]$. While the role of MRI in the diagnosis of acute myocarditis is well established, with recently published recommendations by an expert committee ("Lake Louise Criteria") [27], the role of MRI in chronic myocarditis is less well defined, but is probably important because patients with DCM secondary to chronic myocarditis may show a favourable response to immunomodulatory therapy. De Cobelli et al. [54] reported in patients with biopsy-proven chronic myocarditis a similar focal pattern of enhancement as in patients with acute myocarditis in up to $70 \%$ of a group of patients with chronic inflammation at endomyocardial biopsy. Moreover,
Fig. 8 Extensive transmural anteroapical infarction in 46year-old man. LGE MRI in the vertical long axis (a). Enddiastolic SSFP cine MRI in the horizontal long-axis in the first week (b), at 1 year (c), and at 5 years post-infarction $(\mathbf{d})$. Note the progressive increase in LV end-diastolic volume with thinning of the LV wall, reflecting adverse ventricular remodelling
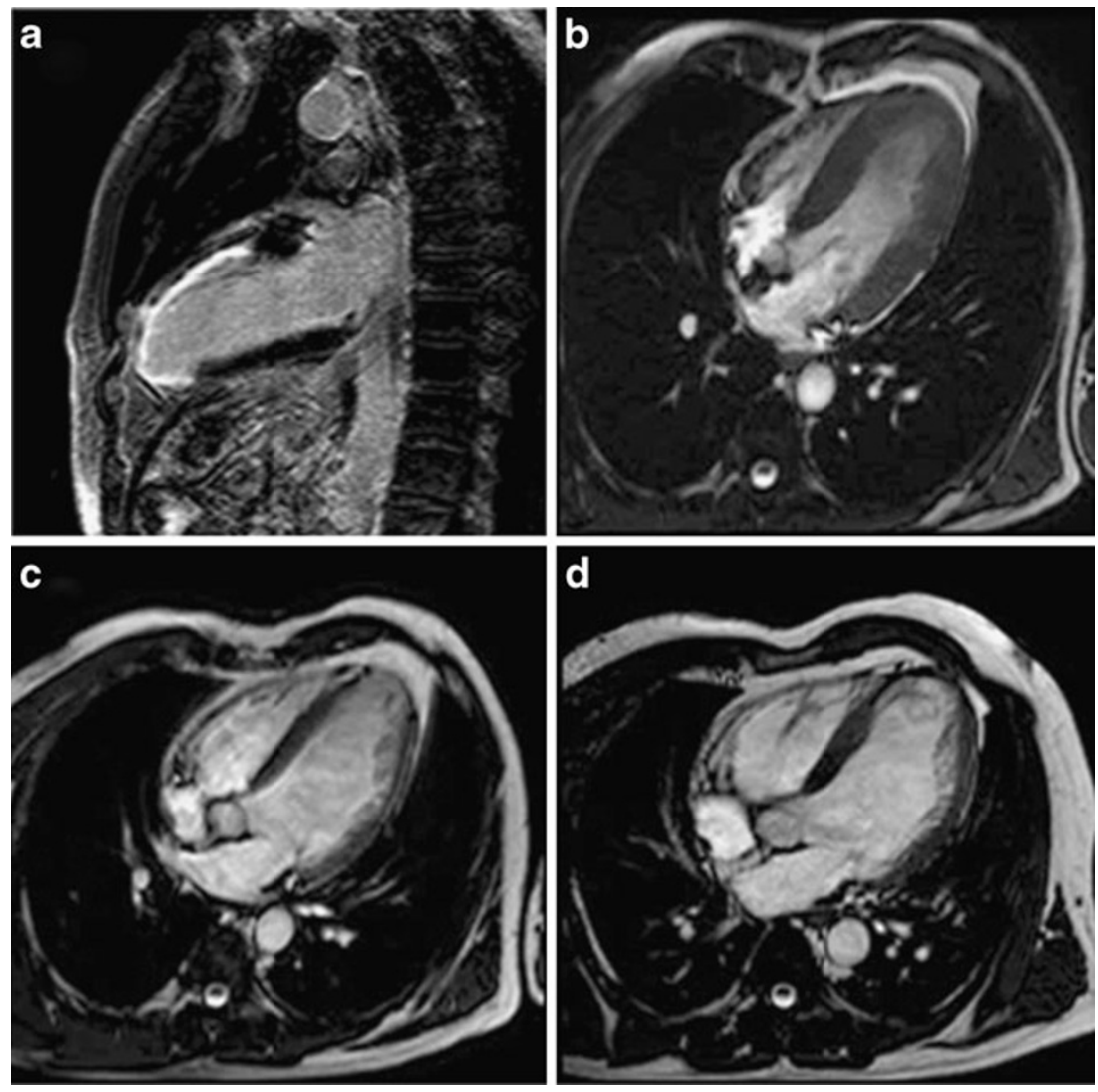
patients with persistent chronic myocarditis frequently showed generalised myocardial oedema and increase in global myocardial enhancement. On the other hand, focal myocardial LGE, probably reflecting myocardial scarring, had low sensitivity and specificity in depicting chronic myocarditis [28, 55]. Finally, Mahrholdt et al. [53] evaluated patients with acute myocarditis at the 3-month follow-up and reported a significant reduction in the extent of midwall/subepicardial focal myocardial enhancement representing residual inflammation or fibrotic scarring.

\section{Left ventricular non-compaction cardiomyopathy}

In DCM patients, myocardial trabeculations, along the LV free wall, often appear more prominent than in healthy subjects [56]. The exact mechanism is unclear but may represent a compensatory phenomenon. Differentiation between DCM and left ventricular non-compaction cardiomyopathy (LVNC) may be challenging, especially in mild forms of LVNC (Fig. 9) [57, 58]. Nowadays recognised as a distinct cardiomyopathy, LVNC is thought to be caused by intrauterine arrest of the process of compaction of the loosely interwoven meshwork of myocardial fibres [59, 60]. As a result, LVNC patients present with prominent trabeculations, deep intertrabecular recesses and a thin epicardial compacta. An alternative hypothesis suggests that the prominent trabeculations represent an adaptive mechanism to compensate for abnormally contracting myocardium [61]. Most commonly, the apical and midventricular part of the inferior and lateral LV wall are affected (Fig. 10) [62]. Attempts to define diagnostic morphological criteria for LVNC have been shown to be challenging, mainly due to the lack of an easy-to-use parameter enabling the differentiation of LVNC patients from normal subjects or other disease entities [61-63]. In brief, criteria have been defined taking into account the two-layered appearance of the myocardial wall, the ratio of non-compacted to compacted myocardium, the number of visible trabeculations apically to the papillary muscles, and the presence of deep intertrabecular spaces visualised on colour Doppler imaging. In a recent study by Kohli and co-workers, it was shown that these diagnostic criteria are likely too sensitive with up to a quarter of heart failure patients and $8 \%$ of control subjects fulfilling one or more criteria for LVNC $[64,65]$. On the other hand, other papers, including MRI findings, suggest that the echocardiographic criteria might be too strict, and that techniques such as MRI may enhance detection of more subtle forms of LVNC [60, 66, 67]. The superior spatial and contrast resolution of MRI makes this technique appealing for the detection of LVNC. The location and extent of the trabecular network, and the
Fig. 9 Dilated dysfunctional LV in a 19-year-old woman. SSFP cine MRI in the horizontal long axis at end-diastole (a) and endsystole (b), and in the vertical long-axis at end-diastole (c) and end-systole (d). The LV enddiastolic volume is $260 \mathrm{ml}$ with an ejection fraction of $11 \%$. Note an extreme thinning of the myocardial wall, in particular of the interventricular septum, and the presence of thick pronounced trabeculations in the LV apex. Although MRI enables the morphological abnormalities to be depicted accurately, it remains challenging in this patient to differentiate between an LV non-compaction cardiomyopathy and a DCM with compensatory trabecular hypertrophy
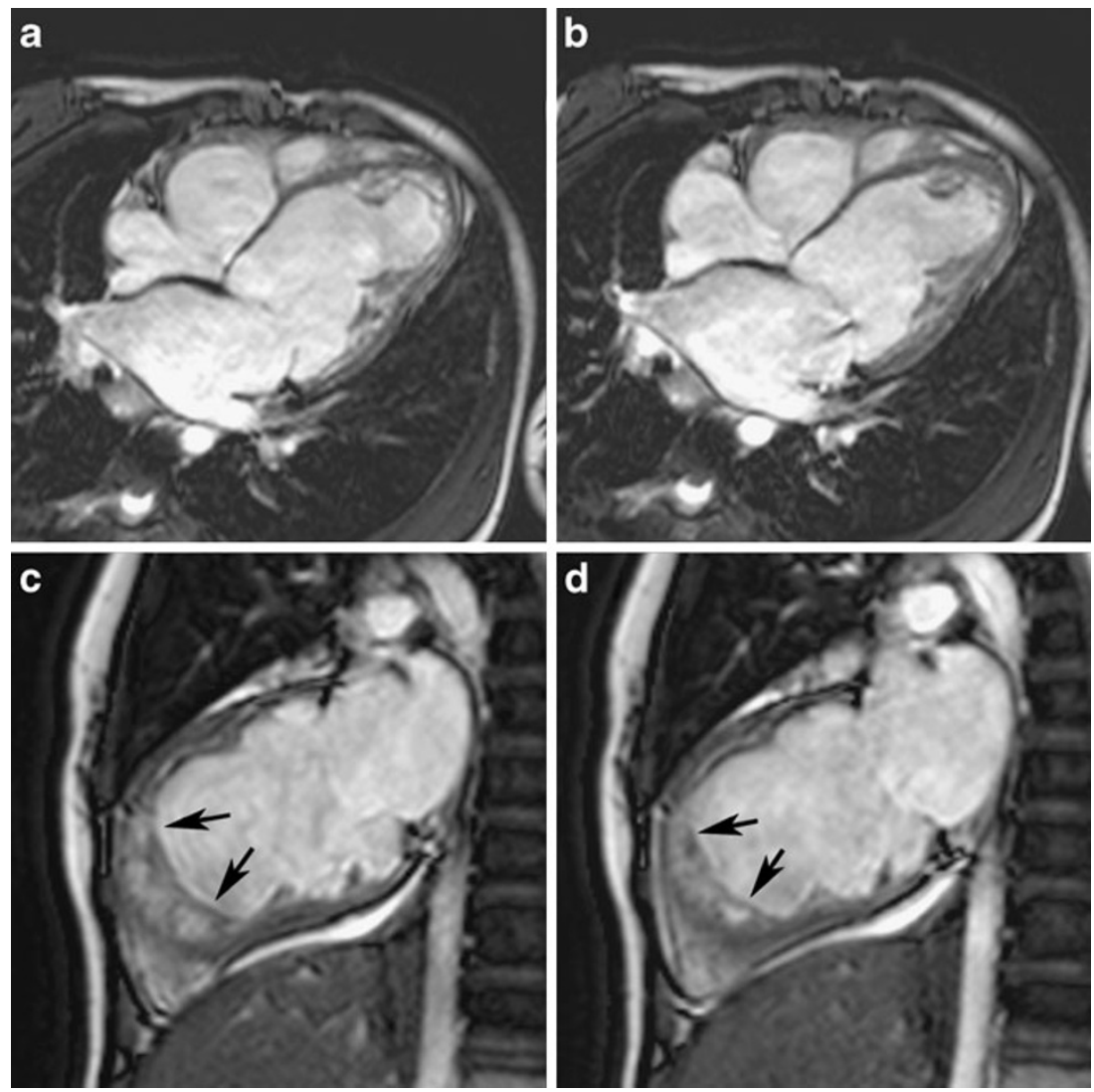


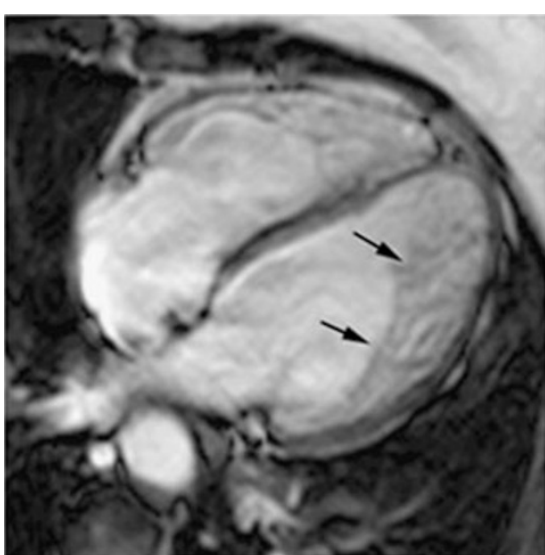

Fig. 10 Typical example of LV non-compaction cardiomyopathy. Four-chamber SSFP cine MR image at end-diastole. Note the presence of an increased number of trabeculations along the LV lateral wall and LV apex (arrowheads)

thickness of the trabeculations and compacta can be well visualised using a combination of cine MRI in different cardiac imaging planes. In addition, these sequences allow the impact of morphological abnormalities on regional and global function to be assessed. LGE MRI may reveal underlying myocardial disease such as replacement fibrosis [68, 69]. In 2005, Petersen et al. [70] established MRI criteria for the diagnosis of LVNC, similar to the abovementioned echocardiographic criteria. An $\mathrm{NC} / \mathrm{C}$ ratio $>2.3$ in diastole distinguished LVNC from normals, athletes and a series of cardiac diseases with a sensitivity and specificity of 86 and $99 \%$ respectively. Jacquier et al. [71] proposed to quantify the percentage of trabeculated LV myocardium, and reported in normals $12 \pm 5 \%$ trabeculated myocardium vs $32 \pm 10 \%$ in LVNC patients. Although promising, the applicability of the above MRI criteria in daily clinical practice needs further confirmation.

\section{Myocardial viability assessment}

Obstructive CAD may cause myocardial ischaemia and dysfunction, and initiate compensatory ventricular remodelling with progressive dilatation, which ultimately may lead to ischaemic heart failure (Fig. 8). The crucial question to solve in these patients is whether percutaneous or surgical coronary revascularisation will improve function in the dysfunctional regions and ultimately improve patient outcome [72]. As the myocardial substrate underlying the dysfunction in the setting of CAD is heterogeneous, including stunned, ischaemic, hibernating, necrotic and scarred myocardium, the goal of myocardial viability assessment is to determine the ischaemic substrate. It is important to emphasise that different ischaemic substrates can be present within the same coronary perfusion territory
$[73,74]$. Only the viable substrates may recover function following reperfusion. Even if there are not yet any prospectively controlled studies on the effects of revascularisation, there is a substantial amount of clinical evidence that patients with reversible LV dysfunction may benefit from a revascularisation procedure $[75,76]$.

The role of MDCT in these patients is mainly focused on visualisation of coronary artery plaques, but no information is provided regarding the viability of the myocardium downstream of the coronary atherosclerotic plaque. MRI is nowadays emerging as one of the preferential techniques for characterising the ischaemic substrate and determining myocardial viability. Three different approaches, or a combination of approaches, can be used for MRI myocardial viability assessment, i.e. end-diastolic wall thickness, contractility reserve and scar imaging (Fig. 11). First, measurement of end-diastolic wall thickness relies on the premise that infarction with subsequent scarring leads to wall thinning, and the magnitude of wall thinning is related to the degree of infarct transmurality [77, 78]. A wall thickness of $6 \mathrm{~mm}$ has been proposed as a cut-off to differentiate between non-viable and viable segments. This approach has excellent sensitivity (95\%, range $94-100 \%)$, but poor specificity $(41 \%$, range $19-53 \%)$ for predicting functional recovery [76-79]. Patients may present "preserved" segmental wall thickness but not recover function following revascularisation, which is probably caused by the presence of subendocardial scarring in this segment. For this reason, the use of the thickness of the non-enhanced rim on LGE MRI may be superior to end-diastolic wall thickness in predicting recovery [80]. Contractile reserve assessment, using low-dose dobutamine stress MRI is the second approach to assessing myocardial viability. Whereas the contractility of normal viable myocardium increases during dobutamine stress, the response of ischaemic, dysfunctional myocardial segments depends on the myocardial substrate (Fig. 12) [78, 81-83]. This approach has good specificity $(83 \%$, range $70-95 \%)$ but moderate sensitivity (74\%, range 50-89\%) [68-71]. The third approach relies on the use of LGE MRI to depict the presence and transmural extent of myocardial scarring. Lack of myocardial LGE in dysfunctional myocardium is indicative of viability, and these segments have a high probability of functional recovery post-revascularisation. On the other hand, the probability of functional recovery is inversely related to the transmural extent of myocardial LGE $[84,85]$. Despite excellent sensitivity $(95 \%$, range $91-$ $99 \%)$, LGE MRI has low specificity (45\%, range $37-54 \%$ ). In particular, for subendocardial infarcts, LGE MRI does not provide information on whether the non-enhanced epicardial rim contains normal, viable or jeopardised myocardium [79]. In order to increase diagnostic accuracy, an integrated MRI approach can be used. For instance, 


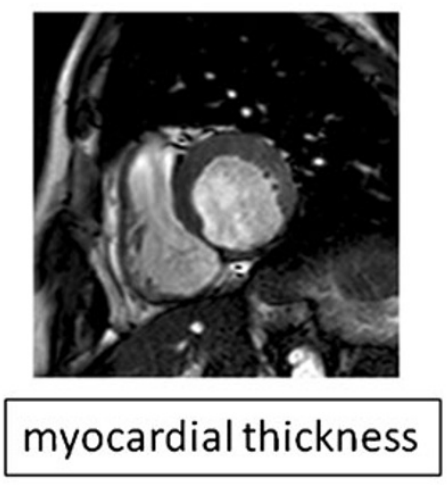

necrosis<smiles>[CH]=C</smiles>

fibrosis-scar<smiles>[CH]=C</smiles>

myocardial wall thinning

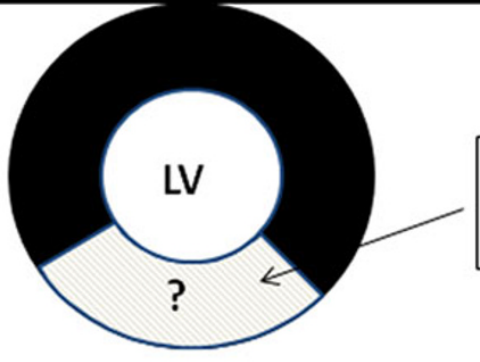

dysfunctional myocardium chronicischemia
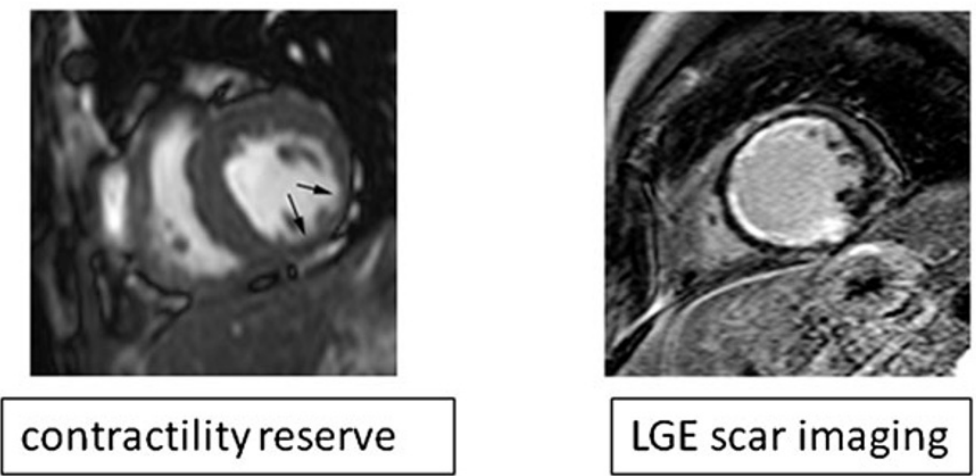

LGE scar imaging

inotropic

response

scar

transmurality<smiles>[CH]=C</smiles>

indicator

probability

of viability
Fig. 11 Different MRI approaches to assessing myocardial viability. Above in this figure is shown a short-axis slice through the left ventricle. The black myocardium represents normal, viable myocardium while the

adding low-dose dobutamine stress MRI in a second step after LGE MRI may be helpful in determining the myocardial substrate of the non-enhanced epicardial rim. As mentioned above, determining the thickness of the nonenhanced epicardial rim may provide additional value regarding viability [80] with a cut-off value of $3 \mathrm{~mm}$ yielding good diagnostic accuracy for the differentiation between viable and non-viable segments.

\section{Arrhythmogenic substrate}

As sudden cardiac death due to ventricular arrhythmias may be the first clinical manifestation of DCM, identification of patients at risk who may benefit from implantable cardioverter defibrillator (ICD) implantation or from an ablation procedure is of primordial importance. Several papers have shown that non-ischaemic cardiomyopathy patients with white myocardium represents chronic dysfunctional myocardium. Three approaches or a combination of approaches can be used to determine the myocardial substrate underlying the chronic dysfunction

midwall myocardial LGE involving more than $25 \%$ of wall thickness are at high risk at inducible ventricular tachycardia and should be referred for definitive anti-arrhythmic device therapy [86-89]. LGE MRI adds predictive value especially in the DCM patients with a mildly to moderately decreased ejection fraction, those with abnormal myocardial enhancement having potential benefit from prophylactic ICD placement [90]. In a recent study by Hombach et al. [10] that included 141 DCM patients, midwall myocardial LGE was not an independent prognostic factor, stressing the need for large prospective studies on this topic [10]. Bogun et al. used LGE MRI to plan an appropriate mapping and ablation strategy in a small group of DCM patients [91]. The location of the scar (endocardial versus epicardial) was an important factor in determining the optimal approach to ablation. However, the success of catheter ablation was low in patients with a scar located in the midwall. 


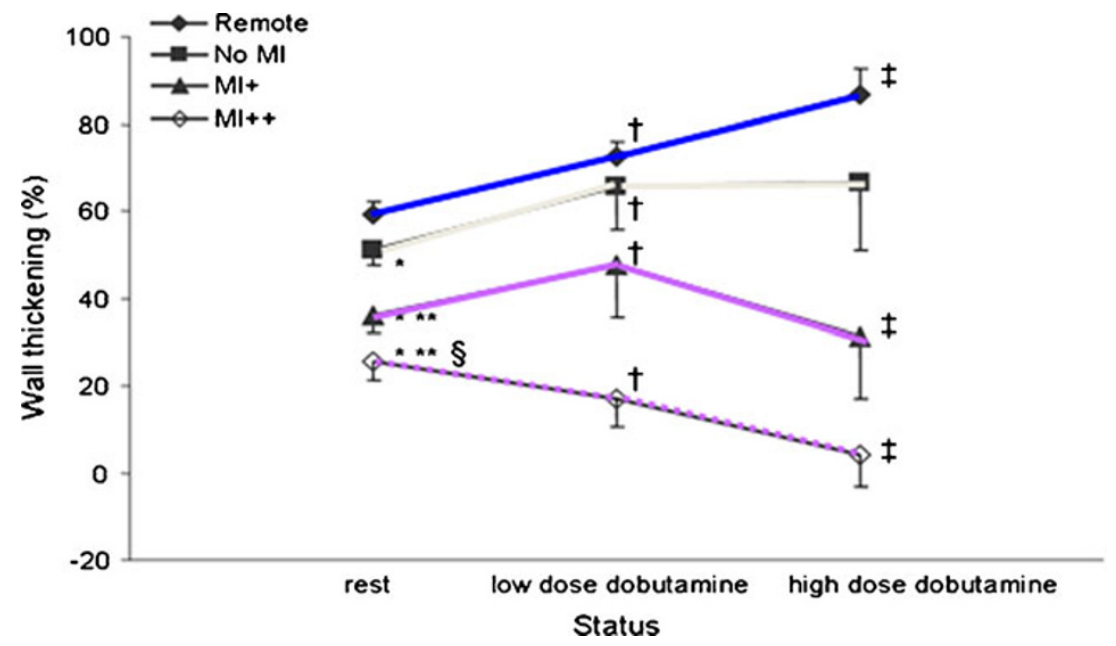

Fig. 12 Relation between myocardial substrate and functional response during low-dose and high-dose dobutamine stress in an animal model with chronic myocardial ischaemia. Whereas contractility gradually increases with increasing doses of dobutamine in normal, remote myocardium, the response to stress is blunted in

\section{Cardiac resynchronisation therapy}

In patients with DCM, ventricular dilatation and replacement fibrosis lead to a heterogeneous excitation spread across the LV wall with a delay in intraventricular conduction and a left bundle branch block morphology on the ECG. Segmental wall motion analysis shows hypokinesis to dyskinesis with a variable degree of dyssynchrony [92, 93]. Ventricular dyssynchrony worsens systolic performance, impedes ventricular filling, and causes paradoxical septal motion during early systole. Cardiac resynchronisation therapy (CRT) consists of the implantation of a biventricular pacemaker in order to improve synchronicity of myocardial contraction leading to improved ventricular performance. In properly selected patients, CRT implantation is associated with improvement of symptoms and a decrease in mortality and hospitalisation for heart failure. However, up to $40 \%$ of CRT-treated patients show no benefit from CRT, urging the need for better identification of responders to CRT treatment.

MRI is a promising tool for identifying and selecting patients eligible for CRT [93, 94]. MRI has the advantage of integrating functional/dyssynchrony imaging with morphological and tissue characterisation imaging. Novel MRI techniques such as DENSE (displacement encoding with stimulated echoes) and TVM (tissue velocity mapping) are appealing for the quantification of the degree of dyssynchrony throughout the LV. In addition, LGE MRI enables the depiction of myocardial scar presence and extent. The higher the scar burden, the lower the chance of CRT response [95-97]. Moreover, as reported by Chalil et al. [98] correct placement of CRT leads is crucial. Positioning chronic ischaemic but viable (no $M I$ ) segments. The response to dobutamine stress in segments containing scarred myocardium depends on the degree of transmurality, being worse in segments with a scar transmurality of more than $50 \%(M I++)$ (Ming Wu, adapted from [66])

of the pacing lead in the scarred myocardium is followed by a lack of CRT response. MRI is currently the best technique for guiding the interventional cardiologist to correctly position CRT leads. The lead should be placed in the myocardium displaying the latest activation. On the other hand, scar stimulation by pacing prevents correct impulse transmission, and consequent inhibition of myocardial contractility.

Another clinically relevant application for MRI and MDCT is the identification of coronary venous anatomy (Fig. 13). In particular, MDCT is a fast and very useful technique in CRT therapy planning. As mentioned above, CRT leads should be placed in the area of latest activation. Once this has been identified, visualisation of the vein tributary to that segment is mandatory. If there is no evidence of a coronary vein on MDCT/MRI in these segments, the transvenous approach is not recommended because it would result in an erroneous lead placement. In these cases, a surgical approach with epicardial lead positioning is preferable. The efficacy of MDCT in depicting coronary venous anatomy, including anatomical variants, is excellent, showing good agreement with anatomical studies and invasive venography $[99,100]$.

\section{Coronary artery imaging}

As previously mentioned, the ventricular dilatation associated with systolic dysfunction can be due to DCM or secondary to coronary artery disease. In the last two decades, coronary artery imaging by MRI and MDCT has been extensively studied as an alternative to invasive catheter angiography. 
Fig. 13 MDCT with volumerendered images (a) and MR coronary angiography (b) showing the heart and the coronary artery and venous system (arrowheads). Ao aorta, $D$ diagonal branch, $L A D$ left anterior descending coronary artery, $L C x$ left circumflex coronary artery, $L M$ left main coronary artery, $R C A$ right coronary artery
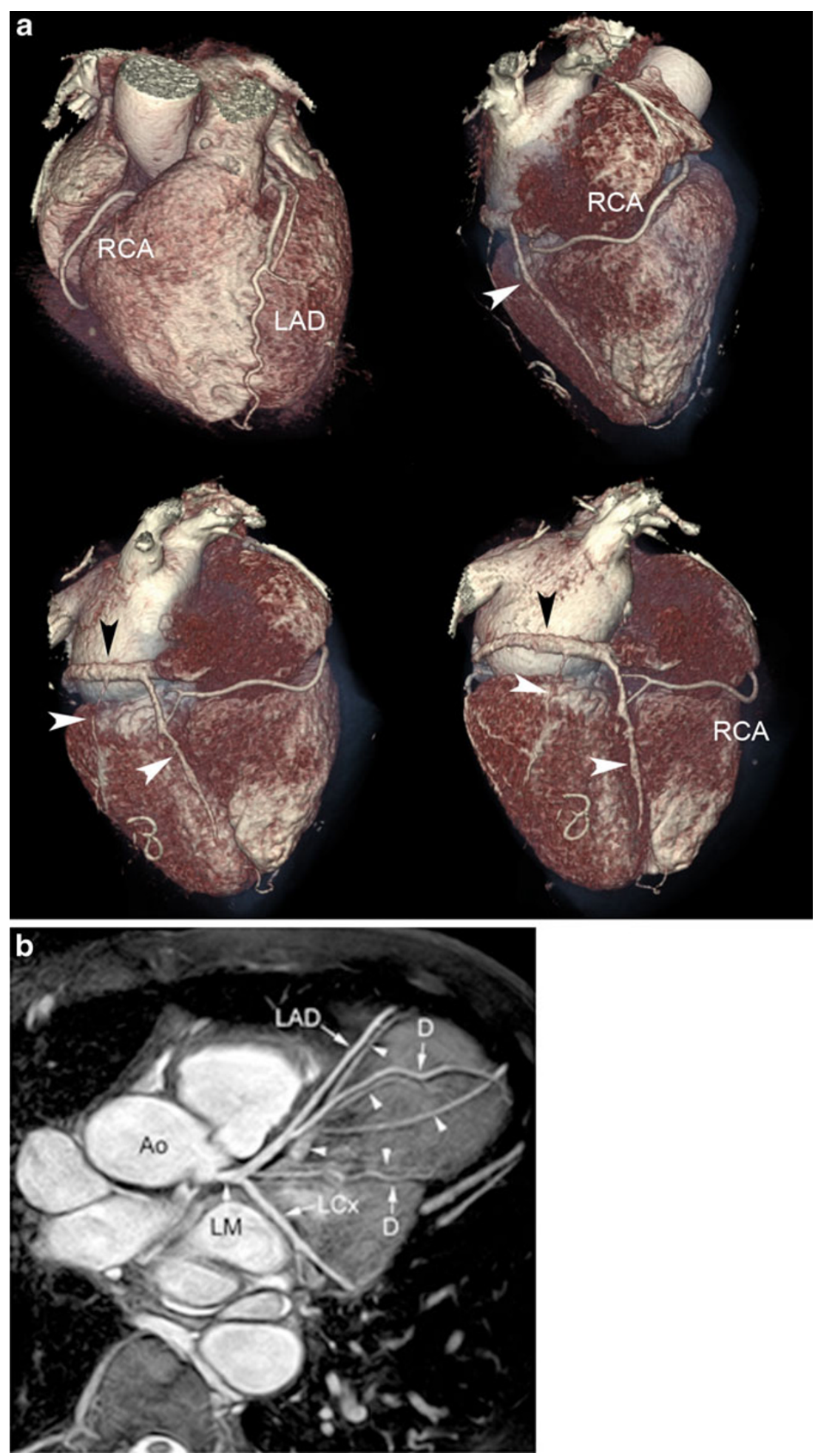

Despite high initial expectations, it has nowadays become evident that MRI has a limited role in depicting coronary artery plaques [101]. Supported by encouraging results of several single-centre and multicentre trials, MDCT is now considered a reliable method for the detection and, in particular, for the exclusion of CAD [12, 102]. However, it should be emphasised that MDCT still faces many challenges, in particular imaging of heavily calcified plaques and stent imaging, although further improvement can be expected with newer generation equipment [103]. 


\section{Conclusions}

In the end, the role of an imaging method is to provide accurate information for the clinician, to minimise the degree of uncertainty in the diagnosis and patient management, and to improve patient outcome. MRI is becoming generally accepted as an important imaging technique in patients with DCM offering the clinician not only accurate information regarding the severity of ventricular dilatation and dysfunction but also regarding myocardial tissue composition, which is important in establishing the underlying cause, in predicting the risk of future events, and in selecting eligible candidates for CRT. The fast and continuous progress in MDCT technology has enabled accurate information regarding coronary artery and venous anatomy to be provided, but this technique has the potential to offer a broader cardiac assessment, including tissue characterisation and functional assessment.

\section{References}

1. Elliott P, Andersson B, Arbustini E et al (2008) Classification of the cardiomyopathies: a position statement from the European Society of Cardiology working group on myocardial and pericardial diseases. Eur Heart J 29:270-276

2. Jefferies JL, Towbin JA (2010) Dilated cardiomyopathy. Lancet 375:752-762

3. Charron P, Arad M, Arbustini E et al (2010) Genetic counseling and testing in cardiomyopathies: a position statement of the European Society of Cardiology Working Group on Myocardial and Pericardial Diseases. Eur Heart J 31:2715-2726

4. Dec GW, Fuster V (1994) Idiopathic dilated cardiomyopathy. N Engl J Med 331:1564-1574

5. Fujita N, Duerinckx AJ, Higgins CB (1993) Variation in left ventricular regional wall stress with cine magnetic resonance imaging: normal subjects versus dilated cardiomyopathy. Am Heart J 125:1337-1345

6. Beltrami CA, Finato N, Rocco M et al (1995) The cellular basis of dilated cardiomyopathy in humans. J Mol Cell Cardiol 27:291-305

7. de Leeuw N, Ruiter DJ, Balk AH (2001) Histopathologic findings in explanted heart tissue from patients with end-stage idiopathic dilated cardiomyopathy. Transpl Int 14:299-306

8. Hughes SE, McKenna WJ (2005) New insights into the pathology of inherited cardiomyopathy. Heart 91:257-264

9. Sun JP, James KB, Yang XS et al (1997) Comparison of mortality rates and progression of left ventricular dysfunction in patients with idiopathic dilated cardiomyopathy and dilated versus nondilated right ventricular cavities. Am J Cardiol 80:1583-1587

10. Hombach V, Merkle N, Torzewski J et al (2009) Electrocardiographic and cardiac magnetic resonance imaging parameters as predictors of a worse outcome in patients with idiopathic dilated cardiomyopathy. Eur Heart J 30:2011-2018

11. Leschka S, Alkadhi H, Plass A et al (2005) Accuracy of MSCT coronary angiography with 64-slice technology: first experience. Eur Heart J 26:1482-1487

12. Budoff MJ, Dowe D, Jollis JG et al (2008) Diagnostic performance of 64-multidetector row coronary computed tomographic angiography for evaluation of coronary artery stenosis in individuals without known coronary artery disease: results from the prospective multicenter ACCURACY (Assessment by Coronary Computed Tomographic Angiography of Individuals Undergoing Invasive Coronary Angiography) trial. J Am Coll Cardiol 52:1724-1732

13. Dewey M, Zimmermann E, Deissenrieder F et al (2009) Noninvasive coronary angiography by 320-row computed tomography with lower radiation exposure and maintained diagnostic accuracy: comparison of results with cardiac catheterization in a head-to-head pilot investigation. Circulation 120:867-875

14. Mark DB, Berman DS, Budoff MJ et al (2010) ACCF/ACR/ AHA/NASCI/SAIP/ SCAI/SCCT 2010 expert consensus document on coronary computed tomographic angiography: a report of the American College of Cardiology Foundation Task Force on Expert Consensus Documents. Circulation 121:2509-2543

15. Semelka RC, Tomei E, Wagner S et al (1990) Interstudy reproducibility of dimensional and functional measurements between cine magnetic resonance studies in the morphologically abnormal left ventricle. Am Heart J 119:1367-1373

16. Buser PT, Wagner S, Auffermann W et al (1990) Threedimensional analysis of the regional contractility of the normal and the cardiomyopathic left ventricle using cine-magnetic resonance imaging. Z Kardiol 79:573-579

17. Gaudio C, Tanzilli G, Mazzarotto P et al (1991) Comparison of left ventricular ejection fraction by magnetic resonance imaging and radionuclide ventriculography in idiopathic dilated cardiomyopathy. Am J Cardiol 67:411-415

18. Strohm O, Schulz-Menger J, Pilz B et al (2001) Measurement of left ventricular dimensions and function in patients with dilated cardiomyopathy. J Magn Reson Imaging 13:367-371

19. MacGowan GA, Shapiro EP, Azhari H et al (1997) Shortening in the fiber and cross-fiber directions in the normal human left ventricle and in idiopathic dilated cardiomyopathy. Circulation 96:535-541

20. Rademakers FE, Marchal G, Mortelmans L et al (2003) Evolution of regional performance after an acute anterior myocardial infarction in humans using magnetic resonance tagging. J Physiol 546:777-787

21. Abdel-Aty H, Boyé P, Zagrosek A et al (2005) Diagnostic performance of cardiovascular magnetic resonance in patients with suspected acute myocarditis. Comparison of different approaches. J Am Coll Cardiol 45:1815-1822

22. Tanner MA, Galanello R, Dessi C et al (2007) A randomized, placebo-controlled, double-blind trial of the effect of combined therapy with deferoxamine and deferiprone on myocardial iron in thalassemia major using cardiovascular magnetic resonance. Circulation 115:1876-1884

23. Kirk P, Roughton M, Porter JB et al (2009) Cardiac T2* magnetic resonance for prediction of cardiac complications in thalassemia major. Circulation 120:1961-1968

24. Simonetti OP, Kim RJ, Fieno DS et al (2001) An improved MR imaging technique for the visualization of myocardial infarction. Radiology 218:215-223

25. Mahrholdt H, Wagner A, Judd RM et al (2005) Delayed enhancement cardiovascular magnetic resonance assessment of non-ischaemic cardiomyopathies. Eur Heart J 26:1461-1474

26. Iles L, Pfluger H, Phrommintikul A et al (2008) Evaluation of diffuse myocardial fibrosis in heart failure with cardiac magnetic resonance contrast-enhanced T1 mapping. J Am Coll Cardiol 52:1574-1580

27. Friedrich MG, Sechtem I, Schulz-Menger J et al (2009) Cardiovascular magnetic resonance in myocarditis: a JACC white paper. J Am Coll Cardiol 53:1475-1487

28. Gutberlet M, Spors B, Thoma T et al (2008) Suspected chronic myocarditis at cardiac MR: diagnostic accuracy and association 
with immunohistologically detected inflammation and viral persistence. Radiology 246:401-409

29. Jerosch-Herold M, Sheridan DC, Kushner JD et al (2008) Cardiac magnetic resonance imaging of myocardial contrast uptake and blood flow in patients affected with idiopathic or familial dilated cardiomyopathy. Am J Physiol Heart Circ Physiol 295:H1234-H1242

30. Flett AS, Hayward MP, Ashworth MT et al (2010) Equilibrium contrast cardiovascular magnetic resonance for the measurement of diffuse myocardial fibrosis: preliminary validation in humans. Circulation 122:138-144

31. Mollet NR, Dymarkowski S, Volders W et al (2002) Visualization of ventricular thrombi with contrast-enhanced MRI in patients with ischemic heart disease. Circulation 106:2873-2876

32. Bogaert J, Taylor AM, Van Kerckhove F et al (2004) Use of inversion-recovery contrast-enhanced MRI technique for cardiac imaging: spectrum of diseases. AJR Am J Roentgenol 182:609-615

33. Bogaert J, Francone M (2009) Cardiovascular magnetic resonance in pericardial diseases. J Cardiovasc Magn Reson 11:14

34. de Roos A, Kroft LJ, Bax JJ et al (2006) Cardiac applications of multislice computed tomography. Br J Radiol 79:9-16

35. Juergens KU, Fischbach R (2006) Left ventricular function studied with MDCT. Eur Radiol 16:342-357

36. Schroeder S, Achenbach S, Bengel F et al (2008) Cardiac computed tomography: indications, applications, limitations, and training requirements: report of a Writing Group deployed by the Working Group Nuclear Cardiology and Cardiac CT of the European Society of Cardiology and the European Council of Nuclear Cardiology. Eur Heart J 29:531-556

37. Abadi S, Roguin A, Engel A et al (2010) Feasibility of automatic assessment of four-chamber cardiac function with MDCT: initial clinical application and validation. Eur J Radiol 74:175-181

38. Taylor AJ, Cerqueira M, Hodgson JM et al (2010) ACCF/ SCCT/ ACR/ AHA/ ASE/ ASNC/ NASCI/ SCAI/ SCMR 2010 appropriate use criteria for cardiac computed tomography: a report of the American College of Cardiology Foundation Appropriate Use Criteria Task Force, the Society of Cardiovascular Computed Tomography, the American College of Radiology, the American Heart Association, the American Society of Echocardiography, the American Society of Nuclear Cardiology, the North American Society for Cardiovascular Imaging, the Society for Cardiovascular Angiography and Interventions, and the Society for Cardiovascular Magnetic Resonance. J Am Coll Cardiol 56:1864-1894

39. Wu YW, Tadamura E, Yamamuro M et al (2008) Estimation of global and regional cardiac function using 64-slice computed tomography: a comparison study with echocardiography, gatedSPECT and cardiovascular magnetic resonance. Int J Cardiol 128:69-76

40. Thilo C, Hanley M, Bastarrika $G$ et al (2010) Integrative computed tomographic imaging of cardiac structure, function, perfusion, and viability. Cardiol Rev 18:219-229

41. Vural M, Uçar O, Selvi NA et al (2010) Assessment of global left ventricular systolic function with multidetector CT and 2D echocardiography: a comparison between reconstructions of 1$\mathrm{mm}$ and 2-mm slice thickness at multidetector CT. Diagn Interv Radiol 16:236-240

42. Bello D, Shah DJ, Farah GM (2003) Gadolinium cardiovascular magnetic resonance predicts reversible myocardial dysfunction and remodeling in patients with heart failure undergoing $\beta$ blocker therapy. Circulation 108:1945-1953

43. Koito H, Suzuki J, Ohkubo N et al (1996) Gadoliniumdiethylenetriamine pentaacetic acid enhanced magnetic resonance imaging of dilated cardiomyopathy: clinical significance of abnormally high signal intensity of left ventricular myocardium. J Cardiol 28:41-49
44. Wu E, Judd RM, Vargas JD et al (2001) Visualization of the presence, location and transmural extent of healed Q-wave and non-Q-wave myocardial infarction. Lancet 357:21-28

45. McCrohon JA, Moon JCC, Prasad SK et al (2003) Differentiation of heart failure related to dilated cardiomyopathy and coronary artery disease using gadolinium-enhanced cardiovascular magnetic resonance. Circulation 108:54-59

46. Soriano CJ, Ridocci F, Estornell J et al (2005) Noninvasive diagnosis of coronary artery disease in patients with heart failure and systolic dysfunction of uncertain etiology, using late gadolinium-enhanced cardiovascular magnetic resonance. J Am Coll Cardiol 45:743-748

47. Nikolaou K, Knez A, Sagmeister S et al (2004) Assessment of myocardial infarctions using multidetector-row computed tomography. J Comput Assist Tomogr 28:286-292

48. Wagner A, Schulz-Menger J, Dietz R et al (2003) Long-term follow-up of patients with acute myocarditis by magnetic resonance imaging. Magma 16:17-20

49. Han Y, Peters DC, Dokhan B et al (2009) Shorter difference between myocardium and blood optimal inversion time suggests diffuse fibrosis in dilated cardiomyopathy. J Magn Reson Imaging 30:967-972

50. Sueyoshi E, Sakamoto I, Uetani M (2010) Contrast-enhanced myocardial inversion time at the null point for detection of left ventricular myocardial fibrosis in patients with dilated and hypertrophic cardiomyopathy: a pilot study. AJR Am J Roentgenol 194:293-298

51. Knaapen P, Götte MJW, Paulus WJ et al (2006) Does myocardial fibrosis hinder contractile function and perfusion in idiopathic dilated cardiomyopathy? PET and MR imaging study. Radiology 240:380-388

52. Moreo A, Ambrosio G, De Chiara B et al (2009) Influence of myocardial fibrosis on left ventricular diastolic function: noninvasive assessment by cardiac magnetic resonance and echo. Circ Cardiovasc Imaging 2:437-443

53. Mahrholdt H, Goedecke C, Wagner A et al (2004) Cardiovascular magnetic resonance assessment of human myocarditis. A comparison to histology and molecular biology. Circulation 109:1250 1258

54. De Cobelli F, Pieroni M, Esposito A et al (2006) Delayed gadolinium-enhanced cardiac magnetic resonance in patients with chronic myocarditis presenting with heart failure or recurrent arrhythmias. J Am Coll Cardiol 47:1649-1654

55. Voigt A, Elgeti T, Durmus T et al (2011) Cardiac magnetic resonance imaging in dilated cardiomyopathy in adults - towards identification of myocardial inflammation. Eur Radiol 21:925935

56. Imai H, Kumai T, Sekiya M et al (1992) Left ventricular trabeculae evaluated with MRI in dilated cardiomyopathy and old myocardial infarction. J Cardiol 22:83-90

57. Richardson P, McKenna W, Bristow M et al (1996) Report of the 1995 World Health Organization/International Society and Federation of Cardiology Task Force on the Definition and Classification of Cardiomyopathies. Circulation 93:841-842

58. Maron BJ, Towbin JA, Thiene G et al (2006) Contemporary definitions and classification of the cardiomyopathies: an American Heart Association Scientific Statement from the Council on Clinical Cardiology, Heart Failure and Transplantation Committee; Quality of Care and Outcomes Research and Functional Genomics and Translational Biology Interdisciplinary Working Groups; and Council on Epidemiology and Prevention. Circulation 113:1807-1816

59. Elshershari H, Okutan V, Celiker A (2001) Isolated noncompaction of the ventricular myocardium. Cardiol Young 11:472-475

60. Pignatelli RH, McMahon CJ, Dreyer WJ et al (2003) Clinical characterization of left ventricular noncompaction in children. A 
relatively common form of cardiomyopathy. Circulation 108:2672-2678

61. Captur G, Nihoyannopoulos P (2010) Left ventricular noncompaction: genetic heterogeneity, diagnosis and clinical course. Int J Cardiol 140:145-153

62. Oechslin EN, Attenhofer Jost CH, Rojas JR et al (2000) Longterm follow-up of 34 adults with isolated left ventricular noncompaction: a distinct cardiomyopathy with poor prognosis. J Am Coll Cardiol 36:493-500

63. Belanger AR, Miller MA, Donthireddi UR et al (2008) New classification scheme of left ventricular noncompaction and correlation with ventricular performance. Am J Cardiol 102:92-96

64. Kohli SK, Pantazis AA, Shah JS et al (2008) Diagnosis of leftventricular non-compaction in patients with left-ventricular systolic dysfunction: time for a reappraisal of diagnostic criteria? Eur Heart J 29:89-95

65. Anderson RH (2008) Ventricular non-compaction - a frequently ignored finding? Eur Heart J 29:10-11

66. McCrohon JA, Richmond DR, Pennell DJ et al (2002) Isolated noncompaction of the myocardium. A rarity or missed diagnosis? Circulation 106:e22-e23

67. Borreguero LJJ, Corti R, de Soria RF et al (2002) Diagnosis of isolated noncompaction of the myocardium by magnetic resonance imaging. Circulation 105:e177-e178

68. Dodd JD, Holmvang G, Hoffmann U et al (2007) Quantification of left ventricular noncompaction and trabecular delayed hyperenhancement with cardiac MRI: correlation with clinical severity. AJR Am J Roentgenol 189:974-980

69. Alsaileek AA, Syed I, Seward JB et al (2008) Myocardial fibrosis of left ventricle: magnetic resonance imaging in noncompaction. J Magn Reson Imaging 27:621-624

70. Petersen SE, Selvanayagam JB, Francis JM et al (2005) Differentiation of athlete's heart from pathological forms of cardiac hypertrophy by means of geometric indices derived from cardiovascular magnetic resonance. J Cardiovasc Magn Reson 7:551-558

71. Jacquier A, Thuny F, Jop B et al (2010) Measurement of trabeculated left ventricular mass using cardiac magnetic resonance imaging in the diagnosis of left ventricular noncompaction. Eur Heart J 31:1098-1104

72. Underwood SR, Bax JJ, vom Dahl J et al (2004) Imaging techniques for the assessment of myocardial hibernation. Report of a Study Group of the European Society of Cardiology. Eur Heart J 25:815-836

73. Wu M, Bogaert J, D'hooge J et al (2010) Closed-chest animal model of chronic coronary artery stenosis. Assessment with magnetic resonance imaging. Int J Cardiovasc Imaging 26:299308

74. Wu M, D'hooge J, Ganame J et al (2010) Non-invasive characterization of the area-at-risk using magnetic resonance imaging in chronic ischemia. Cardiovasc Res 89:166-174

75. Bax JJ, Visser FC, Poldermans D et al (2001) Time course of functional recovery of stunned and hibernating segments after surgical revascularization. Circulation 104:I314-I318

76. Schinkel AF, Bax JJ, Poldermans D et al (2007) Hibernating myocardium: diagnosis and patient outcomes. Curr Probl Cardiol $32: 375-410$

77. Baer FM, Smolarz K, Jungehulsing M et al (1992) Chronic myocardial infarction: assessment of morphology, function, and perfusion by gradient echo magnetic resonance imaging and 99mTc-methoxyisobutyl-isonitrile SPECT. Am Heart J 123:636645

78. Baer FM, Voth E, Schneider C et al (1995) Comparison of lowdose dobutamine-gradient-echo magnetic resonance imaging and positron emission tomography with $[18 \mathrm{~F}]$ fluorodeoxyglucose in patients with chronic coronary artery disease. A functional and morphological approach to the detection of residual myocardial viability. Circulation 91:1006-1015

79. Kaandorp TAM, Lamb HJ, van der Wall EE et al (2005) Cardiovascular MR to assess myocardial viability in chronic ischaemic LV dysfunction. Heart 91:1359-1365

80. Kühl HP, van der Weerdt A, Beek A et al (2006) Relation of enddiastolic wall thickness and the residual rim of viable myocardium by magnetic resonance imaging to myocardial viability assessed by fluorine-18 deoxyglucose positron emission tomography. Am J Cardiol 97:452-457

81. Dendale PAC, Franken RP, Waldmann GJ et al (1995) Lowdosage dobutamine magnetic resonance imaging as an alternative to echocardiography in the detection of viable myocardium after acute infarction. Am Heart J 130:134-140

82. Dendale P, Franken PR, van der Wall EE et al (1997) Wall thickening at rest and contractile reserve early after myocardial infarction: correlation with myocardial perfusion and metabolism. Coron Artery Dis 8:259-264

83. Senior R, Lahiri A (1995) Enhanced detection of myocardial ischemia by stress dobutamine echocardiography utilizing the "biphasic" response of wall thickening during low and high dose dobutamine infusion. J Am Coll Cardiol 26:26-32

84. Kim RJ, Wu E, Rafael A et al (2000) The use of contrastenhanced magnetic resonance imaging to identify reversible myocardial dysfunction. N Engl J Med 343:1445-1453

85. Ramani K, Judd RM, Holly TA (1998) Contrast magnetic resonance imaging in the assessment of myocardial viability in patients with stable coronary artery disease and left ventricular dysfunction. Circulation 98:2687-2694

86. Nazarian S, Bluemke DA, Lardo AC et al (2005) Magnetic resonance assessment of the substrate for inducible ventricular tachycardia in nonischemic cardiomyopathy. Circulation 112:2821-2825

87. Assomull RG, Prasad SK, Lyne J et al (2006) Cardiovascular magnetic resonance, fibrosis, and prognosis in dilated cardiomyopathy. J Am Coll Cardiol 48:1977-1985

88. Wu KC, Weiss RG, Thiemann DR et al (2008) Late gadolinium enhancement by cardiovascular magnetic resonance heralds an adverse prognosis in nonischemic cardiomyopathy. J Am Coll Cardiol 51:2414-2421

89. Shimizu I, Iguchi N, Watanabe H et al (2010) Delayed cardiovascular magnetic resonance as a novel technique to predict cardiac events in dilated cardiomyopathy patients. Int $\mathbf{J}$ Cardiol 142:224-229

90. Lakdawala NK, Givertz MM (2010) Dilated cardiomyopathy with conduction disease and arrhythmia. Circulation 122:527-534

91. Bogun FM, Desjardins B, Good E et al (2009) Delayedenhanced magnetic resonance imaging in nonischemic cardiomyopathy. Utility for identifying the ventricular arrhythmia substrate. J Am Coll Cardiol 53:1138-1145

92. Masci PG, Marinelli M, Piacenti M et al (2010) Myocardial structural, perfusion, and metabolic correlates of left bundle branch block mechanical derangement in patients with dilated cardiomyopathy. A tagged cardiac magnetic resonance and positron emission tomography study. Circ Cardiovasc Imaging 3:482-490

93. Tigen K, Karaahmet T, Kirma C et al (2010) Diffuse late gadolinium enhancement by cardiovascular magnetic resonance predicts significant intraventricular systolic dyssynchrony in patients with non-ischemic dilated cardiomyopathy. J Am Soc Echocardiogr 23:416-422

94. Bleeker GB, Kaandorp TA, Lamb HJ et al (2006) Effect of posterolateral scar tissue on clinical and echocardiographic improvement after cardiac resynchronization therapy. Circulation 113:969-976 
95. Marsan NA, Westenberg JJ, Ypenburg C et al (2009) Magnetic resonance imaging and response to cardiac resynchronization therapy: relative merits of left ventricular dyssynchrony and scar tissue. Eur Heart J 30:2360-2367

96. Aggarwal NR, Martinez MW, Gersh BJ et al (2009) Role of cardiac MRI and nuclear imaging in cardiac resynchronization therapy. Nat Rev Cardiol 6:759-770

97. Leyva F (2010) Cardiac resynchronization therapy guided by cardiovascular magnetic resonance. J Cardiovasc Magn Reson 9:12-64

98. Chalil S, Foley PW, Muyhaldeen SA et al (2007) Late gadolinium enhancement-cardiovascular magnetic resonance as a predictor of response to cardiac resynchronization therapy in patients with ischaemic cardiomyopathy. Europace 9:10311037

99. Sá MI, de Roos A, Westenberg JJ et al (2008) Imaging techniques in cardiac resynchronization therapy. Int J Cardiovasc Imaging 24:89-105
100. Van de Veire NR, Marsan NA, Schuijf JD et al (2008) Noninvasive imaging of cardiac venous anatomy with 64-slice multi-slice computed tomography and noninvasive assessment of left ventricular dyssynchrony by 3-dimensional tissue synchronization imaging in patients with heart failure scheduled for cardiac resynchronization therapy. Am J Cardiol 101:1023-1029

101. Hundley WG, Bluemke DA, Finn JP et al (2010) ACCF/ACR/ AHA/NASCI/SCMR 2010 expert consensus document on cardiovascular magnetic resonance: a report of the American College of Cardiology Foundation Task Force on Expert Consensus Documents. Circulation 121:2462-2508

102. Miller JM, Rochitte CE, Dewey M et al (2008) Diagnostic performance of coronary angiography by 64-row CT. N Engl J Med 359:2324-2336

103. Ehara M, Kawai M, Surmely JF et al (2007) Diagnostic accuracy of coronary in-stent restenosis using 64-slice computed tomography: comparison with invasive coronary angiography. J Am Coll Cardiol 49:951-959 